\title{
Effects of electron collisions on the resistive hose instability in intense charged particle beams propagating through background plasma
}

\author{
Han S. Uhm \\ Department of Molecular Science and Technology, Ajou University, Suwon 442-749, Korea \\ Ronald C. Davidson \\ Plasma Physics Laboratory, Princeton University, Princeton, New Jersey 08543 \\ (Received 18 November 2002; published 25 March 2003)
}

\begin{abstract}
The dispersion relation for the resistive hose instability in a charged particle beam with a flattop density profile is derived from the linearized Vlasov-Maxwell equations. Stability properties of the resistive hose instability where the perturbations are initiated at the beam entrance are investigated. In particular, the complex eigenfrequency $\Omega$ in the dispersion relation is expressed as a function of the real oscillation frequency $\omega$ of the excitation at the beam entrance. As expected, the growth rate $\operatorname{Im} \Omega=\Omega_{i}$ decreases rapidly as the conducting wall approaches the beam $\left(r_{w} / r_{b} \rightarrow 1\right)$. The growth rate also decreases substantially as the frequency ratio $\omega / \nu_{c}$ increases, where $\nu_{c}$ is the electron collision frequency. Stability properties for perturbations propagating through the beam pulse from its head to tail are also investigated. In this case, the growth rate $\operatorname{Im} \omega$ is calculated in terms of the real oscillation frequency $\Omega$ of each beam segment. It is shown that the resonance frequency $\Omega=\Omega_{r}$ corresponding to the infinite growth rate detunes considerably from the betatron frequency $\omega_{\beta}$ of the beam particles. It is also found that the bandwidth corresponding to instability is narrow when the plasma electron collision time $\left(1 / \nu_{c}\right)$ is long compared with the magnetic decay time $\left(\tau_{d}\right)$.
\end{abstract}

DOI: 10.1103/PhysRevSTAB.6.034204

PACS numbers: 29.27.Bd, 41.75.-i, 41.85.-p, 52.40.Mj

\section{INTRODUCTION}

Intense charged particle beams produced in high energy accelerators and transport systems [1-5] have a wide range of applications, including basic scientific research, spallation neutron sources, nuclear waste transmutation, and heavy ion fusion [6-8], to mention a few examples. Charged particle beams propagating through preformed plasma may be subject to various instabilities [9-26] that can cause deterioration of the beam quality. One of the most serious instabilities for a beam propagating through a plasma channel appears to be the resistive hose instability [20-26]. Previous theories [21-26] of the resistive hose instability were developed for highly collisional plasmas, where the electron collision frequency $\nu_{c}$ is much higher than the oscillation frequency $\omega$ of the perturbations, i.e., $\nu_{c} \gg|\omega|$. The electron collision frequency $\nu_{c}$ for an electron temperature $T_{e} \gg 1 \mathrm{eV}$ is about $10^{11} \mathrm{~s}^{-1}$ in ambient air at one atmospheric pressure, where the electron collisions are dominated by electronneutral collisions. Obviously, the electron collision frequency decreases considerably if a plasma channel is preformed in a low-pressure chamber. A typical oscillation frequency $\omega$ of the perturbations is on the order of the transverse betatron frequency $\omega_{\beta}$ of the beam particles in the equilibrium fields, as will be seen later. The betatron frequency of the beam particles is again on the order of the beam-plasma frequency $\omega_{p b}$ or less. Keep in mind that the beam-plasma frequency is determined by the beam density and is independent of the chamber pressure. Therefore, the oscillation frequency $\omega$ may be comparable to or higher than the electron collision frequency $\nu_{c}$ in a preformed plasma channel in a lowpressure chamber. As an example, consider a $1 \mathrm{kA}$ cesium ion beam (mass number $A=137$ ) with average kinetic energy $\left(\gamma_{b}-1\right) m_{b} c^{2}=2.5 \mathrm{GeV}$ corresponding to axial beam velocity $V_{b}=\beta_{b} c=0.2 c$, and beam radius $r_{b}=$ $1 \mathrm{~cm}$. In this case the beam density is calculated to be $n_{b}=10^{12} \mathrm{~cm}^{-3}$, and the corresponding depressed betatron frequency [Eq. (7)] is $\omega_{\beta}=1.5 \times 10^{7} \mathrm{~s}^{-1}$, assuming zero return current $\left(f_{m}=0\right)$. On the other hand, for plasma electron temperature $T_{e}=1 \mathrm{eV}$ and electron density $n_{e}=10^{12} \mathrm{~cm}^{-3}$, the electron collision frequency due to Coulomb collisions is $\nu_{c}=2.9 \times 10^{7} \mathrm{~s}^{-1}$, showing that $\omega_{\beta}$ and $\nu_{c}$ can be comparable in size. In this context, we reinvestigate the resistive hose instability including the important influence of the oscillation frequency $\omega$ of the perturbations, by making use of the Vlasov-Maxwell equations for an infinitely long, intense charged particle beam propagating through a preformed plasma channel. The background plasma is assumed to provide complete neutralization of the beam space charge, and the beam particle motion is assumed to be paraxial $\left(p_{z}^{2} \gg\right.$ $p_{r}^{2}+p_{\theta}^{2}$ ). Moreover, the background plasma may provide partial neutralization of the beam current with fractional current neutralization $f_{m}$. Finally, the present analysis is intended for application to intense, positively charged ion beams propagating through background plasma with density larger than or comparable to the beam density $n_{b}$. In this case, for large-volume plasma, the plasma electrons respond rapidly and provide nearly complete 
neutralization of the ion beam space charge [27]. For applications to intense ion beams for heavy ion beam fusion [28-31], both assisted-pinched transport [28,29] in the target chamber and neutralized ballistic transport $[30,31]$ through background plasma are possible modes for beam propagation. The present analysis is most applicable to assisted-pinched transport since the beam radius is assumed to be approximately constant.

The basic assumptions and theoretical model are presented in Sec. II, where a charged particle beam with a flattop density profile propagates through a background plasma channel. The dispersion relation for the resistive hose instability is derived from the linearized Vlasov-Maxwell equations, assuming long-wavelength $\left(k r_{b} \ll 1\right)$, low-frequency $\left(|\omega| r_{b} \ll c\right)$ perturbations, and that complete charge neutralization is provided by the background plasma, including neutralization of the perturbed beam space-charge field. Here $k$ and $\omega$ are the axial wave number and frequency, respectively, of the perturbations, and $r_{b}$ is the beam radius. When a currentcarrying beam moves through a conducting plasma, its self-magnetic field may follow the beam with a delay time called the magnetic decay time $\tau_{d}$. The magnetic field lines are frozen into the plasma, pulling back the distorted beam segment if the plasma is highly conducting. However, this restoring mechanism, which leads to the resistive hose instability, overshoots and grows due to the motionless resistive plasma medium [21-26]. If the plasma electron collision time $\left(1 / \nu_{c}\right)$ is comparable to or longer than the magnetic decay time $\tau_{d}$, some of the magnetic field lines may slip through the plasma following the beam's transverse motion, thereby weakening the instability mechanism. Here, $\nu_{c}$ is the electron collision frequency. Therefore, the dispersion relation obtained in Sec. II includes the stabilizing influence of a finite magnetic decay time, which can be comparable to or shorter than the electron collision time. The instability driven by the return current is investigated in Sec. III in the limit of a negligibly small value of the magnetic decay time. The instability driven by the return current occurs whenever the fractional current neutralization $f_{m}$ is larger than the critical current $f_{c}$ defined in Eq. (34).

The perturbations are Fourier decomposed according to $\exp [i(k z-\omega t)]=\exp \left[-i\left(\omega \tau+\Omega z / \beta_{b} c\right)\right]$, where $\tau=$ $t-z / \beta_{b} c$ represents the coordinate measured from the head of the beam to the tail, and $\Omega=\omega-k \beta_{b} c$ is the Doppler-shifted frequency seen by a beam particle. Here, $\beta_{b} c$ is the average axial beam velocity. The dispersion relation can be analyzed according to the nature of the initial perturbation. In other words, the beam segment may be treated by using $z$ and $t$ as independent variables, or by using $z$ and $\tau$ as independent variables. If the perturbation is initialized by a deflecting excitation at $z=0$ with oscillation frequency $\omega$, then the complex frequency $\Omega=\omega-k \beta_{b} c$ represents the oscillation and growth (or damping) of the wave as a particular beam segment propagates downstream. Stability properties of the resistive hose instability where the perturbations are excited at the beam entrance are investigated in Sec. IV. In particular, the eigenfrequency $\Omega$ in the dispersion relation is expressed as a function of system parameters and the real oscillation frequency $\omega$ of the excitation at the beam entrance. As expected, the growth rate $\Omega_{i}=$ $\operatorname{Im} \Omega$ decreases significantly as the conducting wall radius $r_{w}$ approaches the beam radius $r_{b}$. The growth rate $\Omega_{i}$ also decreases substantially as the frequency ratio $\omega / \nu_{c}$ increases. However, the real oscillation frequency $\operatorname{Re} \Omega$ is almost independent of the frequency ratio $\omega / \nu_{c}$.

Stability properties for perturbations propagating through the beam pulse from its head to tail are investigated in Sec. V, by selecting $z$ and $\tau$ as independent variables. In this case, the Doppler-shifted frequency $\Omega$ seen by the beam particles scales as the characteristic transverse betatron frequency $\omega_{\beta}$ of the beam particles. In addition, the frequency $\omega$ scales as the magnetic decay time in the plasma channel. The complex eigenfrequency $\omega$ in the dispersion relation is expressed as a function of the real oscillation frequency $\Omega$ of each beam segment. Then $\omega$ determines the growth of the perturbation as one moves backward from the head of the beam. It is shown that the resonance frequency $\Omega=\Omega_{r}$ defined in Eq. (48) and corresponding to infinite growth rate detunes considerably from the betatron frequency $\omega_{\beta}$ of the beam particles. We also show that the instability bandwidth is narrow when the electron collision time $\left(1 / \nu_{c}\right)$ is longer than the magnetic decay time $\left(\tau_{d}\right)$. Finally, it is important to recognize the limitations of the present analysis imposed by the assumption of the flattop density profile. In reality, charged particle beams have a somewhat rounded density profile, which assures a spread in transverse betatron frequencies. Individual beam particles then have a betatron frequency that depends on the transverse energy $[22,23]$. In this case, there is not a distinct resonance frequency, and the maximum growth rate of the instability has a finite value $[22,23]$. We are currently investigating the effects of a rounded density profile and electron collisions on detailed stability behavior, and results of this study will be presented in a future publication.

\section{BASIC ASSUMPTIONS AND THEORETICAL MODEL}

We consider an intense relativistic charged particle beam with radius $r_{b}$ propagating through background plasma, whose conductivity is determined primarily by the plasma electrons. The beam-plasma system is confined transversely within a perfectly conducting cylindrical wall with radius $r_{w}$. Cylindrical polar coordinates $(r, \theta, z)$ are used, with the $z$ axis along the axis of symmetry. In equilibrium, both the beam and plasma are assumed to be azimuthally symmetric $(\partial / \partial \theta=0)$, 
infinitely long, and axially uniform $(\partial / \partial z=0)$. The background plasma, whose density is comparable to or higher than the beam density, is assumed to provide complete neutralization of the beam space charge, and the motion of the beam is assumed to be paraxial $\left(p_{z}^{2} \gg\right.$ $p_{r}^{2}+p_{\theta}^{2}$ ). The beam particles are radially confined by the self-magnetic field produced by the axial current of the beam. Under the assumption that the equilibrium distribution function for the beam particles is axisymmetric and spatially uniform in the axial direction, we recognize that the transverse Hamiltonian and axial momentum of the beam particles are constants of the motion in the equilibrium fields $[5,19,23]$. Therefore, for present purposes, the equilibrium distribution function for the beam particles is taken to be $[5,17]$

$$
F_{b}^{0}\left(H_{\perp b}, p_{z}\right)=\frac{n_{b}}{2 \pi \gamma_{b} m_{b}} \delta\left(H_{\perp b}-T_{\perp b}\right) G_{b}\left(p_{z}\right),
$$

where $n_{b}=$ const is the number density of beam particles on axis $(r=0)$, and $T_{\perp b}$ is a positive constant related to the transverse energy of the beam particles. The quantity $H_{\perp b}$ occurring in Eq. (1) is the transverse Hamiltonian defined by

$$
H_{\perp b}=\frac{1}{2 \gamma_{b} m_{b}} p_{\perp}^{2}+Z_{b} e\left[\Psi_{0}(r)-\Psi_{0 m}\right],
$$

where $Z_{b} e$ is the charge of a beam particle, $\Psi_{0}(r)$ is defined by $\Psi_{0}(r)=-\beta_{b} A_{z}(r), \beta_{b} c$ is the mean axial velocity of the beam particles, $c$ is the speed of light in vacuo, and $A_{z}(r)$ is the axial component of the equilibrium vector potential. In Eq. (2), $r=\left(x^{2}+y^{2}\right)^{1 / 2}$ is the radial distance from the beam axis, and the axial momentum distribution is normalized according to

$$
\int_{-\infty}^{\infty} G_{b}\left(p_{z}\right) d p_{z}=1
$$

Substituting Eq. (1) into

$$
n_{b}^{0}(r)=\int d^{3} p F_{b}^{0}\left(H_{\perp b}, p_{z}\right)
$$

we obtain the step-function (flattop) density profile

$$
n_{b}^{0}(r)= \begin{cases}n_{b}=\mathrm{const}, & 0 \leq r<r_{b} \\ 0, & r_{b}<r \leq r_{w}\end{cases}
$$

for the charged particle beam, where the equilibrium beam radius $r_{b}$ is defined by

$$
r_{b}^{2}=2 \frac{T_{\perp b}}{\gamma_{b} m_{b} \omega_{\beta}^{2}},
$$

and the (depressed) betatron frequency $\omega_{\beta}$ for the beam particles is defined by

$$
\omega_{\beta}^{2}=\beta_{b}^{2} \frac{\omega_{p b}^{2}}{2}\left(1-f_{m}\right) .
$$

The quantity $\omega_{p b}^{2}$ occurring Eq. (7) is the on-axis rela- tivistic beam-plasma frequency squared defined by $\omega_{p b}^{2}=4 \pi n_{b} Z_{b}^{2} e^{2} / \gamma_{b} m_{b}$, and $f_{m}=$ const is the fractional current neutralization. Here, we have assumed that the background plasma carries a constant return current $I_{p}=-f_{m} I_{b}$ in the region $0 \leq r<r_{b}$, where $I_{b}=$ $n_{b} \pi r_{b}^{2} Z_{b} e \beta_{b} c$ is the axial current carried by the beam particles. In equilibrium, the plasma return current, if any, is assumed to have the same radial profile as the beam current. As expected, the (depressed) betatron frequency in Eq. (7) for the beam particles is constant (independent of radial coordinate $r$ ) for the step-function density profile in Eq. (5).

We use the linearized Vlasov-Maxwell equations [5] to investigate the resistive hose instability for perturbations about the intense beam equilibrium described by Eq. (1). We adopt a normal mode approach in which all perturbed quantities are assumed to vary with $\theta, z$, and $t$ according to

$$
\psi(\mathbf{x}, t)=\psi(r) \exp [i(\theta+k z-\omega t)]
$$

where $k$ and $\omega$ are the axial wave number and complex oscillation frequency, respectively, of the perturbations. Note that the perturbations in Eq. (8) have a dipole-mode structure with azimuthal mode number $\ell=1$. In practice, it is convenient to use $\tau$ and $z$, rather than $t$ and $z$, as independent variables, where $\tau=t-z / \beta_{b} c$. In this representation, the perturbations are expressed as

$$
\psi(\mathbf{x}, t)=\psi(r) \exp \left[i\left(\theta-\Omega z / \beta_{b} c-\omega \tau\right)\right]
$$

where $\Omega=\omega-k \beta_{b} c$ is the Doppler-shifted frequency seen by the beam particles. Either $\omega$ or $\Omega$ may be regarded as specified quantities, depending on the situation envisioned. If, for example, a perturbation is initialized by an oscillatory deflecting excitation at $z=0$, then $\omega$ is the real frequency of the excitation, and $\Omega$, generally complex, represents the oscillation and growth (or damping) of the wave as a particular beam segment propagates downstream. If, on the other hand, each beam segment is taken to oscillate at a fixed real frequency $\Omega$, then $\operatorname{Im} \omega$ represents the growth of the wave as one moves backward from the head of the beam. The frequency $\Omega$ occurs through the dynamics of particular beam segments and is expected to scale with the betatron frequency $\omega_{\beta}$, whereas $\omega$ occurs through the magnetic coupling of different beam segments and is expected to scale with the magnetic decay time.

We assume that the background plasma is collisional, although the plasma electrons respond to the perturbed electric field. Therefore, the plasma is characterized by a scalar conductivity $\sigma(r)$, which is primarily contributed by the plasma electrons. We further assume that the perturbed beam space-charge field is completely neutralized by the plasma, which requires that

$$
4 \pi|\sigma(r)| \gg \omega
$$


for $0 \leq r<r_{b}$, where $r_{b}$ is the beam radius. We further consider perturbation wavelengths that are long and frequencies that are low compared with quantities that characterize the beam radius $r_{b}$, i.e.,

$$
\left|k r_{b}\right| \ll 1, \quad\left|\omega r_{b}\right| \ll c,
$$

where $c$ is the speed of light in vacuo. The most unstable modes satisfy Eq. (11) when Eq. (10) holds. It follows that the transverse components of the perturbed fields, $B_{z}, E_{r}$, and $E_{\theta}$, can be neglected, and that the perturbations can be represented in terms of a perturbed axial component of vector potential $A \mathbf{e}_{z}$ according to

$$
\begin{array}{ll}
B_{\theta}(r)=-\frac{d A(r)}{d r}, & B_{r}(r)=\frac{i}{r} A(r), \\
E_{z}(r)=-\frac{1}{c} \frac{\partial A(r)}{\partial t} . &
\end{array}
$$

The plasma electrons respond to the axial component $E_{z}(r)$ of the perturbed electric field in Eq. (12).

After carrying out some algebraic manipulations, Ampere's law for the perturbed axial component of vector potential $A(r)$ can be expressed as

$$
\frac{d}{d r} \frac{1}{r} \frac{d}{d r} r A+\frac{4 \pi i \omega \sigma(r)}{c^{2}} A=-\frac{4 \pi}{c} J_{b}(r),
$$

where the conductivity $\sigma(r)$ is related to the perturbed axial plasma current $J_{p}(r)$ by $J_{p}(r)=\sigma E_{z}(r)$ and the perturbed axial beam current density $J_{b}(r)$ is calculated from

$$
J_{b}(r)=Z_{b} e \int d^{3} p \nu_{z} F_{b} .
$$

The perturbed beam distribution function $F_{b}$ occurring in Eq. (14) is calculated by the method of the character- istics, which can be expressed as $[5,19,23]$

$$
\begin{aligned}
F_{b}(\mathbf{x}, \mathbf{p}, t)= & Z_{b} e G_{b}\left(p_{z}\right) \frac{\partial}{\partial H_{\perp b}} F_{b}^{0}\left(H_{\perp b}\right) \int_{-\infty}^{t} d t^{\prime} \frac{\mathbf{p}_{\perp}^{\prime}}{\gamma_{b} m_{b}} \\
& \cdot \nabla_{\perp} A\left(\mathbf{x}^{\prime}, t^{\prime}\right),
\end{aligned}
$$

where use has been made of Eq. (11).

For a given value of axial wave number $k$, there is generally a discrete set of eigenvalues $\omega$ and eigenfunctions $A(r)$ with different radial mode structures. Our interest here lies in the dipole-mode eigenfunction, which corresponds to a sideways displacement of the beam with a minimum of internal distortion. In the low-frequency limit characterized by

$$
4 \pi r_{b}^{2}|\omega \sigma| / c^{2} \ll 1
$$

the dipole-mode eigenfunction inside the beam is expressed as

$$
A(r) \propto r,
$$

representing a rigid transverse displacement of the uniform beam as well as a self-generated $B_{\theta}$ field. If the term proportional to $\sigma$ in Eq. (13) can be neglected in leading order in accordance with Eq. (16), the eigenfunction in Eq. (17) is a self-consistent solution [17] of the eigenvalue equation (13) in the beam interior. Substituting Eq. (17) into Eq. (15), we can carry out the time integration in Eq. (15) for known particle orbits in the equilibrium field configuration. Defining the perpendicular momentum phase angle $\varphi$ by $p_{x}=p_{\perp} \cos \varphi$ and $p_{y}=p_{\perp} \sin \varphi$, the transverse orbits for the step-function density profiles in Eq. (6) are given by

$$
\begin{aligned}
& x^{\prime}\left(t^{\prime}\right)=\frac{p_{\perp}}{\gamma_{b} m_{b} \omega_{\beta}} \cos \varphi \sin \left[\omega_{\beta}\left(t^{\prime}-t\right)\right]+r \cos \theta \cos \left[\omega_{\beta}\left(t^{\prime}-t\right)\right], \\
& y^{\prime}\left(t^{\prime}\right)=\frac{p_{\perp}}{\gamma_{b} m_{b} \omega_{\beta}} \sin \varphi \sin \left[\omega_{\beta}\left(t^{\prime}-t\right)\right]+r \sin \theta \cos \left[\omega_{\beta}\left(t^{\prime}-t\right)\right],
\end{aligned}
$$

where $\omega_{\beta}$ is the (depressed) betatron frequency defined in Eq. (8). Substituting Eqs. (1), (17), and (18) into Eq. (15) and carrying out the time and momentum integrations $[17,23]$, we obtain the eigenvalue equation

$$
\frac{d}{d r} \frac{1}{r} \frac{d}{d r} r A+\frac{4 \pi i \omega \sigma(r)}{c^{2}} A=\frac{\omega_{p b}^{2} \beta_{b}^{2}}{\Omega^{2}-\omega_{\beta}^{2}} \frac{\delta\left(r-r_{b}\right)}{r} A(r),
$$

where $\Omega$ is the Doppler-shifted frequency defined by $\Omega=$ $\omega-k \beta_{b} c$.

If the plasma conductivity $\sigma(r)$ is constant within the beam and constant outside the beam, the eigenfunctions of Eq. (19) are Bessel functions of order unity, and the dispersion relation connecting $\omega$ and $\Omega$ can be derived in closed form from the boundary conditions $A(0)=$ $A\left(r_{w}\right)=0$, as well as from the matching conditions at $r=$ $r_{b}$. We consider the particular case

$$
\sigma(r)=\frac{1}{1-i \omega / \nu_{c}} \times \begin{cases}\sigma_{p}, & 0 \leq r<r_{b}, \\ \sigma_{1}, & r_{b}<r \leq r_{w},\end{cases}
$$

where $\sigma_{p}$ and $\sigma_{1}$ are the dc plasma conductivities in the two regions, and $\nu_{c}$ is the electron collision frequency. The frequency $\omega$ is of the order of the betatron frequency $\omega_{\beta}$ defined in Eq. (7), which is determined by the beam density. On the other hand, the electron collision frequency $\nu_{c}$ in Eq. (20) is determined by plasma properties. In this context, the parameter $\omega / \nu_{c}$ in Eq. (20) can be larger or smaller than unity, depending on the system parameters. 
For a beam of radius $r_{b}$, with conductivity $\sigma(r)$ given by Eq. (20), the solutions to Eq. (19) can be expressed as

$$
A(r)=A_{\text {in }}(r)=J_{1}\left(\kappa_{p} r\right), \quad 0 \leq r<r_{b}
$$

and

$$
A(r)=A_{\text {out }}(r)=J_{1}\left(\kappa_{p} r_{b}\right) \frac{N_{1}\left(\kappa_{1} r_{w}\right) J_{1}\left(\kappa_{1} r\right)-J_{1}\left(\kappa_{1} r_{w}\right) N_{1}\left(\kappa_{1} r\right)}{J_{1}\left(\kappa_{1} r_{b}\right) N_{1}\left(\kappa_{1} r_{w}\right)-J_{1}\left(\kappa_{1} r_{w}\right) N_{1}\left(\kappa_{1} r_{b}\right)}, \quad r_{b}<r \leq r_{w},
$$

where the parameters $\kappa_{p}$ and $\kappa_{1}$ are defined by

$$
\kappa_{p}^{2}=\frac{4 \pi i \omega \sigma_{p}}{c^{2}\left(1-i \omega / \nu_{c}\right)}
$$

and

$$
\kappa_{1}^{2}=\frac{4 \pi i \omega \sigma_{1}}{c^{2}\left(1-i \omega / \nu_{c}\right)}
$$

Here, $J_{1}(x)$ and $N_{1}(x)$ are the Bessel functions of the first and second kinds, respectively, of order unity. Note that the eigenfunctions $A(r)$ in Eqs. (21a) and (21b) vanish at $r=0$ and $r=r_{w}$ and are continuous at $r=r_{b}$. The remaining boundary condition relating $A_{\text {in }}(r)$ and $A_{\text {out }}(r)$ is obtained by multiplying the eigenvalue equations (21a) and (21b) by $r$, integrating across the surface of the beam from $r=r_{b}(1-\varepsilon)$ to $r=r_{b}(1+\varepsilon)$ and taking the limit $\varepsilon \rightarrow 0_{+}$. We obtain

$$
r_{b}\left(\frac{d}{d r} A_{\text {out }}\right)_{r_{b}}-r_{b}\left(\frac{d}{d r} A_{\text {in }}\right)_{r_{b}}=\frac{\omega_{p b}^{2} \beta_{b}^{2}}{\Omega^{2}-\omega_{\beta}^{2}} A\left(r_{b}\right)
$$

for the remaining boundary condition. Substituting Eqs. (21a) and (21b) into Eq. (23), the dispersion relation for the resistive hose instability can be expressed as

$$
\begin{aligned}
\frac{\omega_{p b}^{2} \beta_{b}^{2}}{\Omega^{2}-\omega_{\beta}^{2}}= & \kappa_{1} r_{b} \frac{J_{1}^{\prime}\left(\kappa_{1} r_{b}\right) N_{1}\left(\kappa_{1} r_{w}\right)-J_{1}\left(\kappa_{1} r_{w}\right) N_{1}^{\prime}\left(\kappa_{1} r_{b}\right)}{J_{1}\left(\kappa_{1} r_{b}\right) N_{1}\left(\kappa_{1} r_{w}\right)-J_{1}\left(\kappa_{1} r_{w}\right) N_{1}\left(\kappa_{1} r_{b}\right)} \\
& -\kappa_{p} r_{b} \frac{J_{1}^{\prime}\left(\kappa_{p} r_{b}\right)}{J_{1}\left(\kappa_{p} r_{b}\right)}
\end{aligned}
$$

where $J_{1}^{\prime}(x)=(d / d x) J_{1}(x)$ and $N_{1}^{\prime}(x)=(d / d x) N_{1}(x)$. Note from Eqs. (22a) and (22b) that the Bessel functions on the right-hand side of Eq. (24) are functions of complex arguments. Therefore, these Bessel functions are also complex functions. Equation (24) can be used to investigate stability properties of the resistive hose instability in a charged particle beam propagating through a background plasma for a broad range of system parameters.

We consider the particular case

$$
|\omega| \sigma_{1} \ll c^{2} / 4 \pi r_{b}^{2}
$$

which assures that magnetic diffusion through the weakly conducting region $r_{b}<r \leq r_{w}$ is fast compared with the time scale $\omega^{-1}$, and therefore the terms in the dispersion relation in Eq. (24) proportional to $\sigma_{1}$ are small. In this case, the dispersion relation in Eq. (24) reduces to

$$
\frac{\omega_{p b}^{2} \beta_{b}^{2}}{\Omega^{2}-\omega_{\beta}^{2}}=-\kappa_{p} r_{b} \frac{J_{1}^{\prime}\left(\kappa_{p} r_{b}\right)}{J_{1}\left(\kappa_{p} r_{b}\right)}-\frac{r_{w}^{2}+r_{b}^{2}}{r_{w}^{2}-r_{b}^{2}},
$$

where $r_{w}$ is the conducting wall radius. Within the context of Eq. (16), we can expand the right-hand side of Eq. (26) in powers of $\kappa_{p} r_{b}$ up to third order. Then, Eq. (26) can be further simplified to give

$$
\frac{\omega_{\beta}^{2} /\left(1-f_{m}\right)}{\Omega^{2}-\omega_{\beta}^{2}}=i \frac{\omega \tau_{d}}{1-i \omega / \nu_{c}}-g,
$$

where the transverse betatron frequency $\omega_{\beta}$ is defined in Eq. (7), the magnetic decay time $\tau_{d}$ for the perturbed current is defined by

$$
\tau_{d}=\frac{\pi \sigma_{p} r_{b}^{2}}{2 c^{2}}
$$

and the geometrical factor $g$ is defined by

$$
g=\frac{1}{1-r_{b}^{2} / r_{w}^{2}} .
$$

In obtaining Eq. (27), use has been made of the definition in Eq. (7). Note that the geometrical factor $g$ in Eq. (29) increases to infinity as the conducting wall radius $r_{w}$ approaches the beam radius $r_{b}$. Equation (27) recovers the previous result [23] for the resistive hose instability in the limit where the electron collision frequency dominates the oscillation frequency of the perturbations, i.e., $\omega / \nu_{c} \rightarrow 0$.

\section{INSTABILITY DRIVEN BY PLASMA RETURN CURRENT}

At moderate values of magnetic decay time $\tau_{d}$, the beam current produces an azimuthal component of the self-magnetic field, and this azimuthal magnetic field cannot move freely through the plasma even if a segment of the beam is displaced sideways. Instead, this field pulls back the displaced beam segment. When this restoring force overshoots, the resistive hose instability [25] develops (Secs. IV and V). On the other hand, if the magnetic decay time $\tau_{d}$ is so short that $|\omega| t_{d} \ll 1$, then Eq. (27) can be approximated by

$$
\frac{\omega_{\beta}^{2}}{\Omega^{2}-\omega_{\beta}^{2}}=-\left(1-f_{m}\right) g .
$$

Solving Eq. (30) for $\Omega^{2}$ gives 


$$
\Omega^{2}=\frac{\left(1-f_{m}\right) g-1}{\left(1-f_{m}\right) g} \omega_{\beta}^{2}
$$

Note from Eq. (31) that $\Omega^{2}<0$ whenever $\left(1-f_{m}\right) g-$ $1<0$, corresponding to one purely growing solution $(\operatorname{Im} \Omega>0)$ and one purely damped $(\operatorname{Im} \Omega>0)$ solution. Therefore, Eq. (31) predicts instability provided the plasma return current satisfies

$$
f_{m}>f_{c}=\frac{g-1}{g}=\frac{r_{b}^{2}}{r_{w}^{2}} .
$$

This unstable mode is called the return-current-driven instability. The beam current and plasma return current flow in opposite directions, thereby generating a repulsive force. Therefore, the beam is displaced transversely by the plasma current. The perturbation is purely growing without any oscillatory motion.

Collisions of course modify the dispersion relation in Eq. (31). For example, in the limit where the oscillation frequency $\omega$ of the perturbation is much larger than the electron collision frequency $\nu_{c}$, we express $i \omega \tau_{d} /(1-$ $\left.i \omega / \nu_{c}\right) \approx-\tau_{d} \nu_{c}$ and the dispersion relation in Eq. (27) can be approximated by

$$
\Omega^{2}=\frac{\left(1-f_{m}\right)\left(g+\nu_{c} \tau_{d}\right)-1}{\left(1-f_{m}\right)\left(g+\nu_{c} \tau_{d}\right)} \omega_{\beta}^{2},
$$

which recovers the previous result in Eq. (31) in the limit where $\tau_{d} \nu_{c} \rightarrow 0$. Note that Eq. (33) predicts instability $\left(\Omega^{2}<0\right)$ whenever $f_{m}>f_{c}$, where $f_{c}$ is defined as

$$
f_{c}=\frac{g+\nu_{c} \tau_{d}-1}{g+\nu_{c} \tau_{d}} \text {. }
$$

On the other hand, whenever $f_{m}<f_{c}$, it follows from Eq. (33) that $\Omega^{2}>0$ and the solutions are purely oscillatory. For a large-volume plasma with $r_{b}^{2} \ll r_{w}^{2}$ and $g \approx$ 1, note from Eq. (34) that the threshold value of the return current for the onset of instability reduces to $f_{c}=$ $\tau_{d} \nu_{c} /\left(1+\tau_{d} \nu_{c}\right)$.

\section{PERTURBATIONS WITH REAL FREQUENCY EXCITATION AT $z=0$}

In this section, we consider an initial excitation of a beam segment at $z=0$ with real oscillation frequency $\omega$. Returning to Eq. (9), it then follows that $\Omega$, which is generally complex, represents the oscillation and growth (or damping) of the perturbation as the beam segment propagates downstream. The characteristic value of $\omega$ could be of the order of $\omega_{\beta}$, which is a natural oscillation frequency of the beam particles. It is convenient to express the dispersion relation in Eq. (27) as $\chi^{2}=\Omega^{2}(1-$ $\left.f_{m}\right) / \omega_{\beta}^{2}=a+i \beta$, where the parameters $\alpha$ and $\beta$ are defined by

$$
\alpha=1-f_{m}-\frac{g+\left(\tau_{d}+g / \nu_{c}\right) \omega^{2} / \nu_{c}}{g^{2}+\left(\tau_{d}+g / \nu_{c}\right)^{2} \omega^{2}}
$$

and

$$
\beta=-\frac{\omega \tau_{d}}{g^{2}+\left(\tau_{d}+g / \nu_{c}\right)^{2} \omega^{2}} .
$$

Expressing $\chi=\chi_{r}+i \chi_{i}$, the normalized growth (damping) rate $\chi_{i}=\operatorname{Im} \chi$ and real frequency $\chi_{r}=\operatorname{Re} \chi$ solving Eq. (27) can be expressed as

$$
\chi_{i}= \pm \frac{1}{\sqrt{2}}\left(\sqrt{\alpha^{2}+\beta^{2}}-\alpha\right)^{1 / 2}
$$

and

$$
\chi_{r}=\mp \frac{\beta}{\sqrt{2}} \frac{1}{\sqrt{\left(\alpha^{2}+\beta^{2}\right)^{1 / 2}-\alpha}},
$$

respectively. The upper sign in Eq. (37) corresponds to instability with $\chi_{i}=\operatorname{Im} \chi>0$.

Equations (35)-(38) can be used to investigate detailed stability properties over a wide range of system parameters. For example, if $\omega \tau_{d} \rightarrow 0$ and $\omega / \nu_{c}$ is finite, then $\beta \rightarrow 0$ and the growth rate of the unstable solution in Eq. (37) reduces to $\chi_{i}=(|\alpha|-\alpha)^{1 / 2} / 2^{1 / 2}$. In this case, $\chi_{i}=0$ whenever $\alpha>0$, corresponding to stable oscillations, whereas $\chi_{i}=|\alpha|^{1 / 2}$ whenever $\alpha<0$, corresponding to instability. For $\omega \tau_{d} \rightarrow 0$, the instability condition $\alpha<0$ reduces directly to the inequality $f_{m}>f_{c}=(1-$ $g) / g$ in Eq. (32) required for the onset of the return current instability, as discussed in Sec. III. Note that the real frequency $\chi_{r}=0$ for $\alpha<0$ and $\omega \tau_{d} \rightarrow 0$, indicating a purely growing instability.

As a second example, we consider Eqs. (35)-(38) for the case $f_{m}=0$ (no return current) and $g=1\left(r_{w}^{2} / r_{b}^{2} \rightarrow\right.$ $\infty)$. In this case, Eqs. (35) and (36) reduce to

$$
\alpha=\frac{\left(\omega \tau_{d}+\omega / \nu_{c}\right) \omega \tau_{d}}{1+\left(\omega \tau_{d}+\omega / \nu_{c}\right)^{2}}
$$

and

$$
\beta=-\frac{\omega \tau_{d}}{1+\left(\omega \tau_{d}+\omega / \nu_{c}\right)^{2}},
$$

respectively. The normalized growth rate for the unstable mode $\left(\chi_{i}>0\right)$ in Eq. (37) is given by

$$
\begin{aligned}
\chi_{i}= & \frac{\left(\omega \tau_{d} / 2\right)^{1 / 2}}{\left[1+\left(\omega \tau_{d}+\omega / \nu_{c}\right)^{2}\right]^{1 / 4}} \\
& \times\left[1-\frac{\left(\omega \tau_{d}+\omega / \nu_{c}\right)}{\left[1+\left(\omega \tau_{d}+\omega / \nu_{c}\right)^{2}\right]^{1 / 2}}\right]^{1 / 2},
\end{aligned}
$$

where $\omega \tau_{d}>0$ and $\omega / \nu_{c}>0$ are assumed without loss of generality. For $\left(\omega \tau_{d}+\omega / \nu_{c}\right)^{2} \ll 1$, note that Eq. (41) reduces to $\chi_{i}=\left(\omega \tau_{d} / 2\right)^{1 / 2}$, which is the familiar normalized growth rate of the resistive hose instability [23]. For $\omega^{2} \tau_{d}^{2} \ll 1$, but values of $\omega / \nu_{c}$ corresponding to $\omega^{2} / \nu_{c}^{2}>1$, we note from Eq. (41) that the effect of electron collisions is to reduce the growth rate relative to the value $\left(\omega \tau_{d} / 2\right)^{1 / 2}$. 
As noted earlier, Eqs. (35)-(38) can be used to investigate detailed stability properties over a wide range of system parameters. Typical results are illustrated in Figs. 1 and 2 for the case where $\omega \tau_{d}=0.075$ and $f_{m}=0$. In Fig. 1, the normalized growth rate $\chi_{i}=\operatorname{Im} \Omega / \omega_{\beta}$ is plotted versus the geometrical factor $g=\left(1-r_{b}^{2} / r_{w}^{2}\right)^{-1}$ for $g$ ranging from 1 to 2 , and several values of the parameter $\omega / \nu_{c}$. Note that $g=1$ corresponds to $r_{w}^{2} / r_{b}^{2} \rightarrow$ $\infty$, whereas $g=2$ corresponds to a nearby conducting wall with $r_{b} \approx 0.7 r_{w}$. As expected, the proximity of a conducting wall greatly reduces the growth rate of the resistive hose instability. Furthermore, the normalized growth rate $\chi_{i}$ decreases for increasing values of $\omega / \nu_{c}$ [see also Eq. (41) for $g=1$ ], although the normalized oscillation frequency $\chi_{r}$ is relatively insensitive to the value of $\omega / \nu_{c}$ (see Fig. 2). Note also from Fig. 1 that the growth rate of the resistive hose instability can be substantial, even when $\omega^{2} \tau_{d}^{2} \ll 1$. For example, from Fig. 1, for $g=1$ and $\omega / \nu_{c}=0.5$, we obtain $\operatorname{Im} \Omega=0.125 \omega_{\beta}$.

As an illustrative example characteristic of heavy ion fusion applications $[7,8,27]$, we consider a $1 \mathrm{kA}$ cesium ion beam corresponding to mass number $A=m_{b} / m_{p}=$ 137. The beam ions are singly charged with $Z_{b}=1$, and the average kinetic energy is $\left(\gamma_{b}-1\right) m_{b} c^{2}=2.5 \mathrm{GeV}$ corresponding to $\beta_{b}=0.2$. Assuming that the beam radius is $r_{b}=1 \mathrm{~cm}$, the beam density is calculated to be $n_{b}=10^{12} \mathrm{~cm}^{-3}$. The corresponding betatron frequency calculated from Eq. (7) is $\omega_{\beta}=1.5 \times 10^{7} \mathrm{~s}^{-1}$, assuming zero return current $\left(f_{m}=0\right)$. The electron collision frequency for Coulomb collisions is given by [32]

$$
\nu_{c}=2.9 \times 10^{-6} n_{e} \ln \Lambda T_{e}^{-3 / 2},
$$

where the typical value of the Coulomb logarithm is about $\ln \Lambda=10$. Assuming the electron temperature is about $T_{e}=1 \mathrm{eV}$ and taking $n_{e}=n_{b}=10^{12} \mathrm{~cm}^{-3}$, the

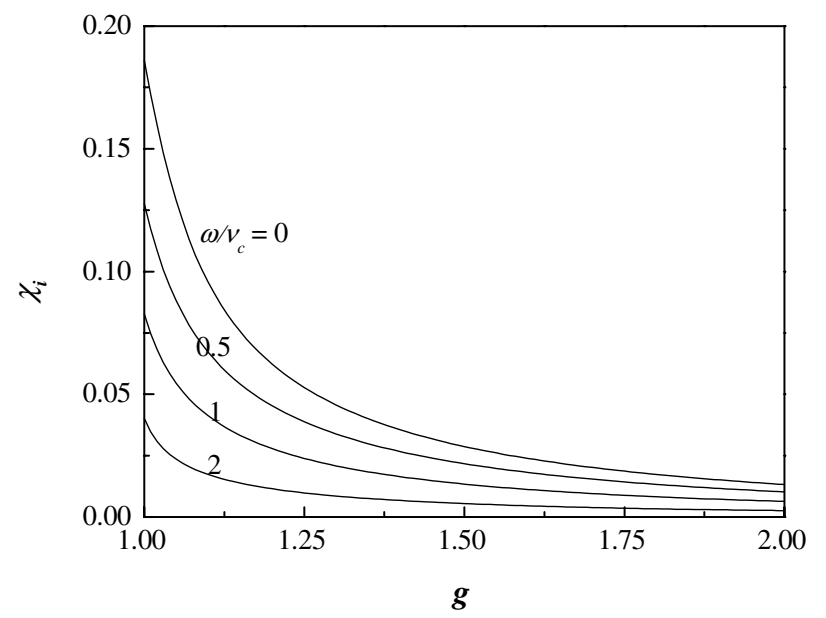

FIG. 1. Plots of the normalized growth rate $\chi_{i}$ versus the geometrical factor $g$ obtained from Eqs. (35)-(37) for several values of the frequency ratio $\omega / \nu_{c}$, and $\omega \tau_{d}=0.075$ and $f_{m}=0$.

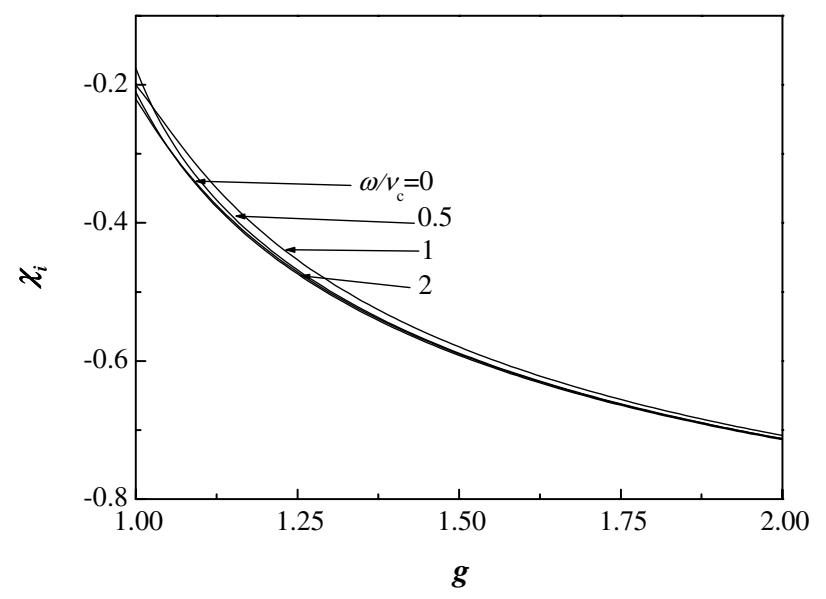

FIG. 2. Plots of the normalized real frequency $\chi_{r}$ versus the geometrical factor $g$ obtained from Eq. (38) for parameters identical to Fig. 1.

electron collision frequency is $\nu_{c}=2.9 \times 10^{7} \mathrm{~s}^{-1}$. The conductivity of the background plasma (assumed fully ionized) can be expressed as [33]

$$
\sigma=\frac{3 m_{e}}{(16 / \sqrt{\pi}) Z e^{2} \ln \Lambda}\left(\frac{2 \kappa T_{e}}{m_{e}}\right)^{3 / 2}
$$

where $\kappa$ is Boltzmann's constant. From Eq. (43), for $T_{e}=$ $1 \mathrm{eV}$ and $\ln \Lambda=10$, the conductivity is $\sigma=3 \times$ $10^{12} \mathrm{~s}^{-1}$. Therefore, the magnetic decay time defined in Eq. (28) is calculated to be $\tau_{d}=5 \times 10^{-9} \mathrm{~s}$. Assuming the characteristic value of real frequency is $\omega=\omega_{\beta}$ at $z=0$, we obtain $\omega \tau_{d}=7.5 \times 10^{-2}$, which is much less than unity. Substituting the appropriate numbers into Eq. (37), the instability growth rate is $\operatorname{Im} \Omega=\Omega_{i}=$ $0.13 \omega_{\beta}$ for $g \rightarrow 1$. The corresponding real oscillation frequency calculated from Eq. (38) is $\operatorname{Re} \Omega=-0.22 \omega_{\beta}$. Note that the growth rate of the resistive hose instability can be a substantial fraction of the betatron frequency of the beam particles for this choice of system parameters.

\section{INSTABILITY FOR PERTURBATIONS PROPAGATING THROUGH THE BEAM PULSE}

A finite size beam pulse is often required to propagate to a target in practical applications. Although the beam head may be at the target, the tail of the beam pulse may deviate from the proposed path due to perturbations that are initiated at the beam head and propagate through the beam pulse, growing during the propagation. In Eq. (9), the coordinate $\tau$ defined by

$$
\tau=t-\frac{z}{\beta_{b} c}
$$

represents the distance (in units of $\beta_{b} c$ ) from the beam head to position $z$. Here, all perturbed quantities are assumed to vary according to $\exp \left[i\left(\theta-\Omega z / \beta_{b} c-\right.\right.$ $\omega \tau)$ ]. If each beam segment in the beam pulse is taken to 
oscillate at a fixed real axial wave number $\Omega / \beta_{b} c$, then $\omega$ in Eq. (9) represents the oscillation and growth (or damping) of the perturbation as one moves backward from the head of the beam.

In the collision-dominated limit where $|\omega| / \nu_{c} \rightarrow 0$, the dispersion relation in Eq. (27) reduces to

$$
i \omega \tau_{d}=g+\frac{\omega_{\beta}^{2} /\left(1-f_{m}\right)}{\Omega^{2}-\omega_{\beta}^{2}},
$$

which determines the dependence of $\omega$ on $\Omega$ (assumed real). Several points are noteworthy from Eq. (45). First, the complex eigenfrequency $\omega$ is purely imaginary for real values of $\Omega$. Moreover, instability ( $\operatorname{Im} \omega>0$ ) occurs only over a bounded range of $\Omega^{2}$ satisfying

$$
\begin{aligned}
& \frac{\omega_{\beta}^{2}}{\left(1-f_{m}\right) g}\left[\left(1-f_{m}\right) g-1\right]<\Omega^{2}<\omega_{\beta}^{2}, \\
& \quad \text { for } f_{m}<1-\frac{1}{g}=\frac{r_{b}^{2}}{r_{w}^{2}}, \\
& 0<\Omega^{2}<\omega_{\beta}^{2}, \quad \text { for } f_{\mathrm{m}}>1-\frac{1}{g}=\frac{r_{b}^{2}}{r_{w}^{2}} .
\end{aligned}
$$

Second, the growth rate $\operatorname{Im} \omega$ approaches (unphysically) infinity as $\Omega^{2}$ approaches the betatron frequency squared from below, i.e., $\Omega^{2} \rightarrow \omega_{\beta}^{2}$. This occurs for the flattop density profile in Eq. (5) for the choice of distribution function in Eq. (1), because all particles in the beam are in resonance with the wave at this frequency. Keep in mind from Eqs. (7) and (18) that all beam particles in a flattop density profile execute transverse oscillations at the betatron frequency $\omega_{\beta}=$ const defined in Eq. (7). Therefore, choosing $\Omega=\omega_{\beta}$ would cause a very strong growth of the perturbation that propagates from beam head to tail. For (more physical) rounded beam profiles, however, the growth rate for the resistive hose instability is finite $[22,23]$ for any value of $\Omega$ because the beam particles in these profiles oscillate with different betatron frequencies determined by their position. Third, Eq. (45) explicitly exhibits the dependence of the growth rate on the current neutralization factor $f_{m}$. The destabilizing effect of $f_{m}$ is due to the repulsive interaction between the beam current and the plasma return current. It is particularly significant that instability can occur even for $\Omega=0$ when $f_{m}>1-1 / g=r_{b}^{2} / r_{w}^{2}$, as evident from Eq. (32). Finally, we note from Eq. (46a) that the range of $\Omega^{2}$ corresponding to instability decreases as the conducting wall approaches the beam, i.e., $g \rightarrow \infty$, indicating stabilization by proximity of the conducting wall.

The full dispersion relation in Eq. (27) can be expressed in the equivalent form

$$
i \omega \tau_{d}=\frac{\left[\left(1-f_{m}\right)\left(\Omega^{2}-\omega_{\beta}^{2}\right) g+\omega_{\beta}^{2}\right] \nu_{c} \tau_{d}}{\left(1-f_{m}\right)\left(\Omega^{2}-\omega_{\beta}^{2}\right)\left(\nu_{c} \tau_{d}+g\right)+\omega_{\beta}^{2}},
$$

which relates the complex eigenfrequency $\omega$ to the real frequency $\Omega$ of the beam segment for arbitrary value of the electron collision frequency $\left(\nu_{c} \tau_{d}\right)$. Equation (47) recovers the result in Eq. (45) in the limit of high collision frequency characterized by $\nu_{c} \tau_{d} \rightarrow \infty$. Equation (47) also predicts that the frequency $\omega$ is purely imaginary for real values of $\Omega$. For the dispersion relation in Eq. (47), instability $(\operatorname{Im} \omega>0)$ occurs over the bounded range of $\Omega^{2}$ satisfying

$$
\frac{\left(1-f_{m}\right) g-1}{\left(1-f_{m}\right) \mathrm{g}} \omega_{\beta}^{2}<\Omega^{2}<\Omega_{r}^{2}=\frac{\left(1-f_{m}\right)\left(g+\nu_{c} \tau_{d}\right)-1}{\left(1-f_{m}\right)\left(g+\nu_{c} \tau_{d}\right)} \omega_{\beta}^{2},
$$

where $f_{m}<1-1 / g=r_{b}^{2} / r_{w}^{2}$ is assumed in Eq. (48). Equation (48) recovers the previous result in Eq. (46a) in the limit of high collision frequency characterized by $\nu_{c} \tau_{d} \rightarrow \infty$. Moreover, the growth rate $\operatorname{Im} \omega$ in Eq. (47) approaches infinity as $\Omega^{2}$ approaches the resonance frequency squared $\Omega_{r}^{2}$ from below, i.e., $\Omega^{2} \rightarrow \Omega_{r}^{2}$. Note that the resonance frequency $\Omega_{r}$ is generally detuned from the betatron frequency $\omega_{\beta}$ of the beam particles. We also note from Eq. (48) that the width of the range $\Omega^{2}$ corresponding to instability is given by

$$
\Delta \Omega^{2}=\frac{\nu_{c} \tau_{d}}{g\left(1-f_{m}\right)\left(g+\nu_{c} \tau_{d}\right)} \omega_{\beta}^{2},
$$

for the case where $\left(1-f_{m}\right) g>1$, or equivalently $f_{m}<1-$ $1 / g=r_{b}^{2} / r_{w}^{2}$. It is evident from Eq. (49) that $\Delta \Omega^{2}$ exhibits a strong dependence on $\nu_{c} \tau_{d}$, and that the instability bandwidth becomes quite narrow for $\nu_{c} \tau_{d} \ll 1$ and $f_{m} \approx 0$. For the case where $\left(1-f_{m}\right) g<1$, the inequality in Eq. (48) is replaced by $0<\Omega^{2}<\Omega_{r}^{2}$ and the corresponding width of the range of $\Omega^{2}$ corresponding to the instability is $\Delta \Omega^{2}=\Omega_{r}^{2}$. Furthermore, if the fractional current neutralization $f_{m}$ satisfies $f_{m}>f_{c}=1-1 /(g+$ $\nu_{c} \tau_{d}$ ) defined in Eq. (34), then $\Omega_{r}^{2}<0$, and Eq. (47) has a purely growing solution $(\operatorname{Im} \omega>0)$ for $\Omega^{2}=0$.

As an illustrative example, we consider a $1 \mathrm{kA}$ cesium ion beam corresponding to mass number $A=m_{b} / m_{p}=$ 137. For the parameters chosen in Sec. IV, the electron collision frequency obtained from Eq. (42) is $\nu_{c}=2.9 \times$ $10^{7} \mathrm{~s}^{-1}$ and the magnetic decay time defined in Eq. (28) is calculated to be $\tau_{d}=5 \times 10^{-9} \mathrm{~s}$, which gives $\nu_{c} \tau_{d}=$ 0.15 . For $f_{m}=0$ and $g \rightarrow 1$, the resonance frequency is $\Omega_{r}=0.36 \omega_{\beta}$, which is considerably downshifted from the betatron frequency $\omega_{\beta}$ of the beam particles. The instability range in Eq. (49) is given by $\Delta \Omega^{2}=0.13 \omega_{\beta}^{2}$, indicating a narrow band of instability.

\section{CONCLUSIONS}

We have investigated properties of the resistive hose instability in a charged particle beam propagating through a preformed plasma channel. The basic assumptions and theoretical model were presented in Sec. II for an intense beam with a flattop density profile. The dispersion relation for the resistive hose instability was 
derived from the linearized Vlasov-Maxwell equations. The return-current-driven instability was investigated in Sec. III in the limit of negligibly small magnetic decay time. The return current instability occurs whenever the fractional current neutralization $f_{m}$ is larger than the critical value $f_{c}$ defined in Eq. (34). The full dispersion relation in Eq. (27) was analyzed according to the nature of the initial perturbation. Stability properties of the resistive hose instability where the perturbations are initiated at the beam entrance were investigated in Sec. IV. In particular, the complex eigenfrequency $\Omega$ was determined in terms of the real oscillation frequency $\omega$ of the deflecting perturbation at the entrance. As expected, the growth rate $\operatorname{Im} \Omega=\Omega_{i}$ decreases rapidly as the conducting wall approaches the beam $\left(r_{w} / r_{b} \rightarrow 1\right)$. The growth rate also decreases substantially as the frequency ratio $\omega / \nu_{c}$ increases. However, the oscillation frequency $\operatorname{Re} \Omega$ is almost independent of the frequency ratio $\omega / \nu_{c}$. Stability properties for perturbations propagating through the beam pulse from its head to tail were investigated in Sec. V. The complex eigenfrequency $\omega$ was expressed as a function of the real oscillation frequency $\Omega$ of each beam segment. It was shown that the resonance frequency $\Omega=\Omega_{r}$ corresponding to infinite growth rate detunes considerably from the betatron frequency $\omega_{\beta}$ of the beam particles. It was also found that the bandwidth corresponding to instability is narrow when the electron collision time $\left(1 / \nu_{c}\right)$ is long compared to the magnetic decay time $\left(\tau_{d}\right)$.

Finally, we conclude this article by pointing out that the present analysis has been carried out for a flattop density profile of beam particles, where all of the beam particles have the same betatron frequency $\omega_{\beta}=$ const for their transverse motion. In this context, the growth rate $\operatorname{Im} \omega$ approaches infinity whenever each beam segment is taken to oscillate at the fixed real frequency $\Omega=$ $\Omega_{r}$ defined in Eq. (48), thereby developing an absolute instability for perturbations propagating through the beam pulse from its head to its tail. In reality, however, charged particle beams have a somewhat rounded density profile, which assures a spread in betatron frequencies. Individual beam particles then have a transverse betatron frequency that depends on the transverse energy [22,23]. In this case, there is not a distinct resonance frequency, and the maximum growth rate of the instability has a finite value. We are currently investigating the resistive hose instability for rounded density profiles, including the influence of electron collisions on stability behavior. Results of this study will be presented in a future publication.

\section{ACKNOWLEDGMENTS}

This research was supported by the Department of Energy.
[1] R. C. Davidson, Physics of Nonneutral Plasmas (Addison-Wesley Publishing Co., Reading, MA, 1990), and references therein.

[2] A.W. Chao, Physics of Collective Beam Instabilities in High Energy Accelerators (John Wiley \& Sons, Inc., New York, 1993).

[3] M. Reiser, Theory and Design of Charged Particle Beams (John Wiley \& Sons, Inc., New York, 1994).

[4] J. D. Lawson, The Physics of Charged-Particle Beams (Oxford Science Publications, New York, 1988).

[5] R. C. Davidson and H. Qin, Physics of Intense Charged Particle Beam in High Energy Accelerator (World Scientific, Singapore, 2001).

[6] R. A. Jameson, in Advanced Accelerator Concepts, edited by J. S. Wurtele, AIP Conf. Proc. No. 279 (AIP, New York, 1993), p. 969.

[7] E. P. Lee and J. Hovingh, Fusion Technol. 15, 369 (1989).

[8] Proceedings of the 1995 International Symposium on Heavy Ion Inertial Fusion, Location, edited by J. J. Barnard, T. J. Fessenden, and E. P. Lee [J. Fusion Eng. Des. 32, 1-620 (1996)], and references therein.

[9] D. G. Koshkarev and P. R. Zenkevich, Part. Accel. 3, 1 (1972).

[10] R.C. Davidson, and H. S. Uhm, J. Appl. Phys. 51, 885 (1980).

[11] R. C. Davidson and H. S. Uhm, Phys. Fluids 21, 60 (1978).

[12] D. Neuffer, E. Colton, D. Fitzgerald, T. Hardek, R. Hutson, R. Macek, M. Plum, H. Thiessen, and T.S. Wang, Nucl. Instrum. Methods Phys. Res., Sect. A 321, 1 (1992).

[13] D. Neuffer and C. Ohmori, Nucl. Instrum. Methods Phys. Res., Sect. A 343, 390 (1994).

[14] M. Izawa, Y. Sato, and T. Toyomasu, Phys. Rev. Lett. 74, 5044 (1995).

[15] J. Byrd, A. Chao, S. Heifets, M. Minty, T.O. Roubenheimer, J. Seeman, G. Stupakov, J. Thomson, and F. Zimmerman, Phys. Rev. Lett. 79, (1997).

[16] E. Keil and B. Zotter, CERN Report No. CERN_ISR-TH/ 71-58, 1971.

[17] R. C. Davidson, H. Qin, P. H. Stoltz, and T. S. Wang, Phys. Rev. ST Accel. Beams 2, 054401 (1999).

[18] R. C. Davidson and H. S. Uhm, Phys. Lett. A 285, 88 (2001).

[19] H. S. Uhm, R. C. Davidson, and I. Kaganovich, Phys. Plasmas 8, 4637 (2001).

[20] E. J. Lauer, R. J. Briggs, T. J. Fessendon, R. E. Hester, and E. P. Lee, Phys. Fluids 21, 1344 (1978).

[21] M. N. Rosenbluth, Phys. Fluids 3, 932 (1960).

[22] E. P. Lee, Phys. Fluids 21, 1327 (1978).

[23] H. S. Uhm and M. Lampe, Phys. Fluids 23, 1574 (1980).

[24] H. S. Uhm and M. Lampe, Phys. Fluids 24, 1553 (1981).

[25] H. S. Uhm, Am. J. Phys. 48, 270 (1980).

[26] R. F. Fernsler, S. P. Slinker, M. Lampe, and R. F. Hubbard, Phys. Plasmas 2, 4338 (1995), and references therein.

[27] I. D. Kaganovich, G. Shvets, E. Startsev, and R.C. Davidson, Phys. Plasmas 8, 4180 (2001).

[28] C. L. Olson, Nucl. Instrum. Methods Phys. Res., Sect. A 464, 118 (2002). 
[29] D. V. Rose, P. F. Ottinger, D. R. Welch, B. V. Oliver and C. L. Olson, Phys. Plasmas 6, 4094 (1999).

[30] D. R. Welch, D. V. Rose, B.V. Oliver, T. C. Genoni, C. L. Olson and S. S. Yu, Phys. Plasmas 9, 2344 (2002).

[31] R. C. Davidson et al., Laser Part. Beams 20, 377 (2002).
[32] Physicist's Desk Reference, edited by Herbert L. Anderson (AIP, New York, 1989) Chap. 18.

[33] N. A. Krall and A.W. Trivelpiece, Principles of Plasma Physics (McGraw-Hill, New York, 1973), Chap. 6. 